\title{
El valor de un testimonio autógrafo: la versión latina de Leonardo Bruni de la Ética nicomaquea en el manuscrito XXV F 10 de la Biblioteca Comunale Santa Maria la Nuova de Monreale*
}

\author{
Montserrat JIMÉNEZ SAN CRISTÓBAL \\ FECYT - Università degli Studi di Pavia \\ mjsancristobal@gmail.com
}

Recibido: 13 de abril de 2012

Aceptado: 4 de junio de 2012

\section{RESUMEN}

En este trabajo se pretende mostrar la importancia del manuscrito XXV F 10 de la Biblioteca Comunale Santa Maria la Nuova de Monreale que contiene la traducción de la Ética a Nicómaco realizada por Leonardo Bruni con una subscripción y correcciones autógrafas. Se ofrece una descripción codicológica completa del manuscrito, un análisis de la copia, de la decoración y de los distintos tipos de intervención del autor.

Palabras clave: Leonardo Bruni. Manuscrito XXV F 10 Monreale. Descripción codicológica. 'Bianchi girari'. Crítica textual.

JIMÉNEZ SAN CRISTÓBAL, M., «El valor de un testimonio autógrafo: la versión latina de Leonardo Bruni de la Ética nicomaquea en el manuscrito XXV F 10 de la Biblioteca Comunale Santa Maria la Nuova de Monreale», Cuad. Fil. Clás. Estud. Lat. 32.1 (2012) 121-144.

The value of an autograph testimony: Leonardo Bruni's latin version of Ethics to Nicomachus in the manuscript XXV F 10 from the Biblioteca Comunale Santa Maria Nuova at Monreale

\begin{abstract}
In this paper we discuss the importance of the manuscript XXV F 10 that is in the Biblioteca Comunale Santa Maria la Nuova in Monreale. In this manuscript it is contained the translation of the Ethics to Nicomachus that was made by Leonardo Bruni; moreover, there is an autograph annotation and corrections. We present a complete codicological description of manuscript, an analisys of the copy and decoration, and the difference ways of author's intervention.
\end{abstract}

Keywords: Leonardo Bruni. Manuscript XXV F 10 Monreale Codicological Description. 'Bianchi girari'. Textual Criticism.

\footnotetext{
* Agradezco a la profesora Mariarosa Cortesi su disponibilidad y las precisas observaciones y sugerencias que sin duda han mejorado y enriquecido este trabajo. Quisiera también mostrar mi agradecimiento a todo el personal de la Biblioteca Comunale Santa Maria la Nuova de Monreale, en especial a Ignazia Ferraro por su inestimable ayuda y afecto; también al profesor Marco Ercoles por su ayuda y paciencia.
} 
JiMÉNEZ SAN CRISTÓBAL, M., «The value of an autograph testimony: Leonardo Bruni’s latin version of Ethics to Nicomachus in the manuscript XXV F 10 from the 'Biblioteca Comunale Santa Maria Nuova' at Monreale», Cuad. Fil. Clás. Estud. Lat. 32.1 (2012) 121-144.

Sumario 1.Introducción. 2. El códice XXV F 10 de la Biblioteca Comunale Santa Maria la Nuova de Monreale. 2.1. Descripción del códice. 2.2. La copia. 2.3. La decoración. 2.4. Correcciones autógrafas de Leonardo Bruni. 2.5. La conservación en Monreale. 3. Conclusiones. 4. Referencias bibliográficas.

\section{INTRODUCCIÓN}

La existencia de un manuscrito autógrafo puede parecer a primera vista un camino fácil para el editor que se enfrenta a la edición de una obra, sin embargo, en palabras de M. Reeve (1994, p.56): «Ciò che c'è di vero nella teoria è che un autografo non è senz'altro autorevole». En realidad, para un editor la 'autografía' es una cuestión menor, pues se está en contacto con el autor en cuanto copista no en cuanto autor y en ese sentido, aunque su valor es incalculable para otros aspectos relacionados con la labor editora, no se aceptarán lecciones no queridas por el autor aunque estén testimoniadas en un autógrafo (Reeve 1994, p.56).

La versión latina de la Ética a Nicómaco realizada por Leonardo Bruni en 14161417 podría considerarse, en cierto modo, como una traducción crítica e incluso se podría hablar de una 'edición' controlada por el autor ${ }^{1}$. Si bien no se ha conservado ningún manuscrito original, ni de autor ${ }^{2}$, Hankins $(2001 ; 2003)$ ha identificado un testimonio, el conservado en la Biblioteca Comunale de Santa Maria la Nuova de Monreale, $\mathrm{XXV} \mathrm{F} 10^{3}$, que puede considerarse 'autógrafo' o al menos 'autoriale' (Chiesa 2002, p.17) ya que ha sido corregido por Leonardo Bruni tal y como reza en la subscriptio al final del códice ${ }^{4}$.

Lectus a me, L. Arretino, sed cursim. Itaque nescio satis emendatus sit, sed emendatus tamen in plurimis.

A pesar de la enorme difusión de sus obras, es notoria la escasez de documentos autógrafos de Leonardo Bruni así como las posibles causas de la misma ${ }^{5}$. Con todo

\footnotetext{
${ }^{1}$ Para los problemas y características de la edición de los textos humanísticos vid. Kristeller (1996) y Perosa (2000).

${ }^{2}$ Cf. Gasparri (1994, pp.3-4) para una primera aproximación a los conceptos de manuscrito 'original', 'de autor' y 'autógrafo'.

${ }^{3}$ Garuffi (1902, p.231 n $\mathrm{n}^{\mathrm{O}}$ 14) ofrece una primera descripción del códice e informa de la subscriptio en el f. $126^{\text {r }}$; Hankins (2001, p.431 n. 12; 2003, p.235 n. 1) amplía esta información en su descripción y señala las correcciones y anotaciones de la mano de Leonardo Bruni. El trabajo de Hankins (2001) se recoge en Hankins (2003) por lo que a partir de ahora citaremos por esta última referencia cuando haya coincidencia.

${ }^{4}$ El Assessorato Regionale dei Beni Culturali e dell'Identità Siciliana ha autorizado la reproducción y la publicación del códice XXV F 10 conservado en la Biblioteca Comunale Santa Maria la Nuova de Monreale (Palermo).

${ }^{5}$ Viti (1992, p.277) señala, por ejemplo en el ámbito epistolar, la escasa atención de Bruni (al menos hasta 1436) por recopilar sus cartas, la costumbre de dictarlas y, en el ámbito literario, la falta de necesidad de conservar los originales o borradores, considerados inútiles una vez concluida y determinada la obra. Por su parte
} 


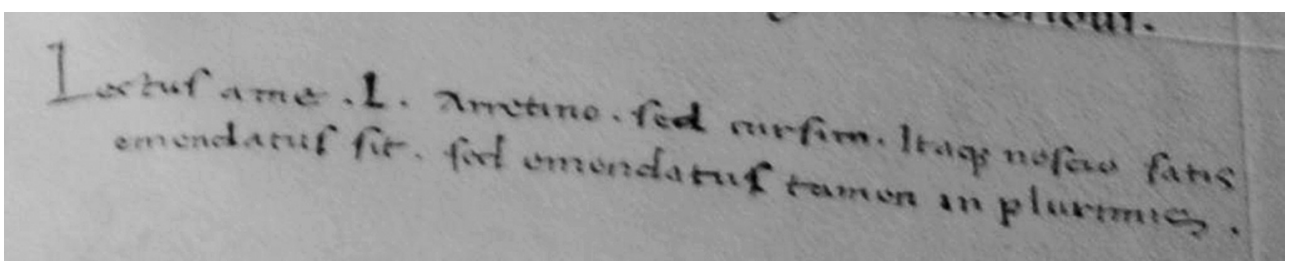

Fig. 1. Monreale, Biblioteca Comunale Santa Maria la Nuova, XXV F 10, f. $126^{\mathrm{r}}$.

parece que los únicos códices literarios en los que hay certeza (y unanimidad por parte de los estudiosos) de la mano latina ${ }^{6}$ de Bruni son el laurenciano Strozzi 44, f. $104^{\mathrm{v}}$, y el que aquí nos ocupa ${ }^{7}$. Hankins $(2003$, p.235) sotiene que no hay razones para dudar de la autenticidad de la subscriptio del códice de Monreale y también Bianca (2002, p.145 n. 66) parece aceptar la paternidad bruniana ${ }^{8}$. Hasta el momento es el único testimonio conocido en el que se constata que la mano de las correcciones y adiciones (interlineales y marginales) coincide con la mano de dicha subscriptio. Es decir, se trata de un ejemplo de la labor de correción que Bruni ejercía sobre las copias de sus obras una vez acabadas y puestas en circulación, la fase 'post-publication' denominada por Hankins $(2001$, p.431) y que hasta el momento se conocía de forma indirecta ${ }^{9}$. El ductus es rígido y está caracterizado, tal y como lo describe Bianca (2002, p.145 y n.66), por la separación de las letras; el uso de dos $s$ minúsculas distintas: una mayúscula pequeña alargada que desciende bajo la línea (normalmente al final de la misma), y otra alta y recta que no desciende bajo la línea; la unión de $c t$. Hay que

Hankins (2001, p.428) apunta que la preferencia a dictar sus traducciones a un amanuense se debiera a una posible «arthritic condition in his hands». En cualquier caso está constatada la preferencia del autor a dictar, práctica frecuente, por otro lado, entre los humanistas del momento (cf., por ejemplo, Rizzo 1984², pp.95-96).

${ }^{6}$ Para la mano griega de Bruni vid. el estado de la cuestión en Viti (1993, pp.294-302 y bibliografía); Berti (1995, pp.284-294 y bibliografía); Bianca (2002, p.144, nn. 63 y 70, y bibliografía).

${ }^{7}$ La mano cancilleresca de Bruni fue identificada por Zaccaria (1998, pp.543-545), por su parte Viti (1992, pp.282-285) identificó la firma de Bruni en documentos notariales (Id., foto $1 \mathrm{a}, \mathrm{b}, \mathrm{c}, \mathrm{d}$, e) y en su testamento (Id. foto 4) publicado por Giustiniani (1964). Frente a esa mano se encontraría la "literary hand" (Hankins 2003, p.235) o textualis (Bianca 2002, p.144 y n. 64) que parece constatada en una nota del Laur. Strozzi 44, f. 104", identificada por Griggio (1986, p.39 y Tav. I) y en la subscriptio del códice de Monreale.

${ }^{8}$ Señala elementos coincidentes entre la subscriptio, la nota del Strozzi 44, f. $104^{\mathrm{v}}$, y una nota atribuida a Bruni en el Plut. 49.7, f. 36 (Bianca 2002, p.145, n. 66 y Tav. 4): el nexo ct, la $s$ final en forma de 5, la separación de las letras.

${ }^{9}$ De la intervención de Bruni en copias individuales nos habla el propio autor, por ejemplo en la Ep.IV 25 (19) a Pileo de Marini (Florencia, 12 de febrero de 1424) en la que explica que ante la dificultad de encontrar copistas para satisfacer al destinatario con las obras que le han sido requeridas ha tenido que comprar un códice a un amigo y lo ha revisado: (...) Quod autem de libris scribundis rogas, non deerit tibi diligentia mea. Verum admirabilis est apud nos eius rei penuria, nam et studiosi permulti sunt et qui mercede scribant admodum pauci. Ego tamen quo tibi morem geram, scrutatus omnia, cum tandem nichil reperirem, exoravi quendam ex familiaribus meis ut libros quosdam sui ipsius gratia quos ille scripserat, venundaret. Sunt autem Ethicorum libri, quos nuper traduxi, et Commentaria primi belli Punici cum quibusdam orationibus Demosthenis et Oeconomicorum libro, sat, ut michi primo aspectu visum est, emendare perscripti. Haec emere licebit (...) (Mehus 1741, I, pp.133134; se ha actualizado la puntuación). Para las cartas de Bruni citadas en este trabajo se sigue la numeración del epistolario según Gualdo Rosa (1980). 


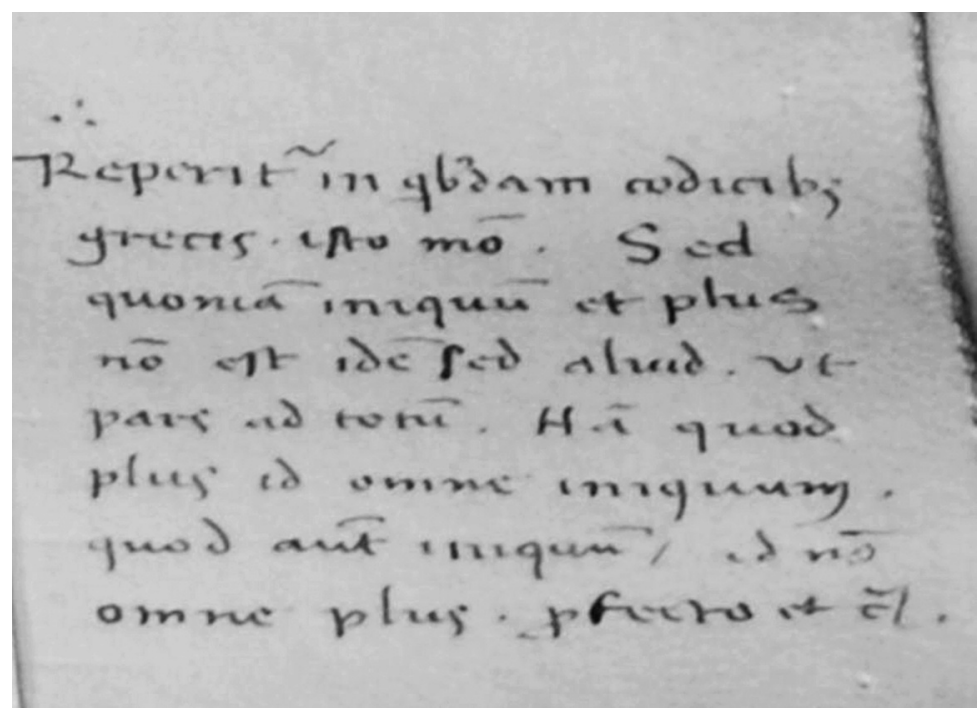

Fig. 2. Monreale, Biblioteca Comunale Santa Maria la Nuova, XXV F 10, detalle margen derecho f. $56^{\mathrm{r}}$.

notar también el empleo de dos $d$ minúsculas: una con trazo recto vertical (o carolina) y otra con el trazo inclinado ligeramente hacia la izquierda; una $b$ con un trazo alto y recto, la unión de st y la $g$ con el lóbulo inferior abierto; la $e$ minúscula caracterizada por un trazo horizontal que sube por encima de la parte derecha del lóbulo, éste casi siempre abierto. Todas estas características pueden apreciarse tanto en la subscripción como en las notas marginales, así por ejemplo la que aparece en el margen externo del f. 56 ${ }^{\mathrm{r}}$, también publicada por Hankins (2003, p.239).

Una vez aceptada la originalidad de la subsciptio y de las correciones, para determinar aún más el valor del manuscrito de Monreale habría que situarlo en el contexto de la tradición manuscrita de la obra que contiene. La difusión de la versión bruniana de la Ética fue excepcional, sobre todo durante el siglo XV: a la inmensa cantidad de copias manuscritas ${ }^{10}$ hay que sumar las ediciones incunables y posteriores ${ }^{11}$. A tal difusión no se ha correspondido, sin embargo, un interés por parte de la comunidad científica ya que hasta el momento no existe ninguna edición crítica.

${ }^{10}$ Lines (2002, p.483) indica 246 pero no aporta catálogo. Por nuestra parte, sólo hemos localizado 236 de entre los que sólo tres no habían sido identificados por Hankins (1997): Londres, British Library, Harl. 3305; Bolonia, Biblioteca Universitaria, ms. 595 T (4); Florencia, Biblioteca Medicea Laurenziana, Ashburnham 953 (884). La mayoría son del siglo XV (sólo hay dos códices del siglo XVI, dos del siglo XVII y uno de los siglos XVII-XVIII).

${ }^{11}$ Según Hankins (2003, p.191) la obra sobrevive en treinta y una "early editions" y Lines (2002, p.483) establece trece para el siglo XVI. En el repertorio de Cortesi-Fiaschi (2008, pp.217-220) se da cuenta de diez ediciones incunables y trece del siglo XVI. Durante el siglo XVII el interés por las obras de Aristóteles disminuyó rápidamente (Schmitt 1983, pp.36-50) y es por ello que las ediciones de la versión latina de Bruni se hacen cada vez más raras. 
De los datos obtenidos a partir de una primera colación del códice de Monreale con un grupo de testimonios fiables de dicha tradición ${ }^{12}$, que garantizan un ámbito cercano al autor (dadas ciertas características codicológicas y textuales, datación, etcétera), se puede afirmar que el monrealense, antes de las correcciones autógrafas, compartía errores comunes y presentaba un texto muy cercano al de otros manuscritos. Sin embargo, a pesar de su posible temprana datación como veremos, no puede ser modelo, al menos modelo único, de ninguno de ellos ya que, después de las correcciones, sigue presentando omisiones que el resto no comparte con él, aunque no será este el lugar para tratar estas cuestiones. A pesar de todo, es un testimonio fundamental para la reconstrucción del texto de la versión latina pues ofrece sin duda la voluntad de su autor, pero a ello se volverá en el parágrafo dedicado a las correcciones de Bruni. Pasemos ahora a los detalles del manuscrito.

\section{EL CÓDICE XXV F 10 DE LA BIBLIOTECA COMUNALE SANTA MARIA LA NUOVA DE MONREALE}

\subsection{DESCRIPCIÓN DEL CÓDICE:}

Biblioteca Comunale Santa Maria la Nuova Monreale (M) ${ }^{13}$

XXV F 10 ${ }^{14}$. Vitela; s. XV 1/4; ff. I (pap.) + I (perg.) + 126 (vit.) + I (pap.); mm. 230 x 175 (172 x 102); los ff. 6, 39, 67, 68, 79, 83, 91, 99 presentan mutilado el margen sin afectar al texto; numeración moderna a lápiz; pautado a punta seca; escritura a página completa, 25 11. por página con disposición destacada; 13 cuadernos distribuidos: $1-12^{10}$ (quiniones), el $13^{6}$ (ternión); encuadernación 'amateur' del siglo XIX (propia de la biblioteca), en piel clara y cartón (hojas de acanto y flores blancas sobre fondo azul); iniciales miniadas 'a bianchi girari' (iniciales en amarillo sobre fondos policromados rojos, azules verdes y racimos entrelazados) de origen florentino (ff. $1^{\mathrm{r}}, 5^{\mathrm{r}}, 18^{\mathrm{r}}, 27^{\mathrm{r}}, 40^{\mathrm{v}}$, $53^{\mathrm{v}}, 67^{\mathrm{r}}, 76^{\mathrm{r}}, 80^{\mathrm{r}}, 102^{\mathrm{r}}, 113^{\mathrm{r}}$ ); marcas de puntizones; escritura redonda humanística; títulos en rojo y en mayúscula; correcciones interlineales y marginalia de Leonardo Bruni, f. $126^{\mathrm{r}}$ subscriptio autógrafa: «Lectus a me L. Arretino sed cursim. Itaque nescio sa-

\footnotetext{
${ }^{12}$ Los testimonios seleccionados para esta colación completa son: el laurenciano Plut. 79.7, copiado por Giovanni Aretino entre 1418 y 1420 para Cosimo de' Medici (Ullman 1960, pp.94; Scarcia 1981, p.137); el conservado en la Biblioteca Nazionale Centrale di Firenze (BNCF), Conv. Soppr. C7 2677, procedente de la Badia florentina que ofrece un texto fiable a pesar de ciertas lagunas (Soudek 1968, p.61) y con mucha probabilidad un texto autorizado por Bruni (Hankins 2003, p.236) y en el que junto al Riccardiano ms. 114 (ca. 14191425), también colacionado, Hankins (2003, p.236) señaló concomitancias con el monrealense en cuanto a las notas marginales se refiere; el Vaticano lat. 2997 (a. 1429), que según Baron (1928, p.75) está emparentado con el laurenciano Plut. 79.10 ( $a .1427$ y copia personal de P.Bracciolini) pero ofrece lecturas mejores. Además hemos cotejado otros treinta códices y las ediciones de Estrasburgo 1469, Valencia 1473-74, Zaragoza 1492 y París 1496-97 en distintos loci.

13 A partir de este momento nos referiremos al códice tanto explícitamente como con la sigla M.

${ }^{14}$ Garufi (1902, p.231) ofrece la signatura XXV. F. 8.10. pero no queda rastro de ella en el códice. Hankins (2003, p.235) ofrece la signatura que aparece en el verso de la hoja de guarda y que es la que actualmente ha sido asignada al códice en el Elenco dei Manoscriti de la biblioteca, cf. Kristeller (1963-1992, I, p.393).
} 
tis emendatus sit, sed emendatus tamen in plurimis»; maniculae posteriores; en el margen superior del verso de los ff. $5^{\mathrm{v}}-20^{\mathrm{v}}$ se indica «Lib.»; en el margen superior del recto de los ff. $6^{\mathrm{r}}-17^{\mathrm{r}}\left\langle\mathrm{I} »\right.$, en el de los ff. $18^{\mathrm{r}}-21^{\mathrm{r}} \ll \mathrm{II} »$, y en el de los ff. $22^{\mathrm{r}}-26^{\mathrm{r}}\langle 2 »$.

F. $\mathrm{I}^{\mathrm{r}}$ guarda (perg.), a lápiz, de mano actual, «62»; tabla del contenido de otra mano del s. XV «i ${ }^{\circ}$ Leonardi Aretini prefatio in libros ethicorum $\mid$ ii $^{\circ}$ Ethicorum Aristotelis liber primus feliciter»; a lápiz, de mano actual, se lee «Monreale».

F. I v guarda (perg.), de mano del s. XVIII o XIX «Ethica Aristotelis latine reddita $\mid$ Codex menbran. sęc. XV».

Ff. $1^{\mathrm{r}}-5^{\mathrm{r}}$ : Praemissio quaedam ad evidentiam novae translationis Ethicorum Aristotelis $\mathrm{f} .1^{\mathrm{r}}$ «Leonardi Aretini Prefatio in libros Ethicorum», inc.: «Aristotelis Ethicorum libros facere latinos... »; f. $5^{\text {r: }}$ «... cum antea non essent. Finis».

Ff. $5^{\mathrm{r}}-126^{\mathrm{v}}$ : Aristoteles, Ethica ad Nicomachum f. $5^{\mathrm{r}}$ «Ethicorum Aristotelis liber pri | mus incipit feliciter.» inc.: «Omnis ars omnisque doct $\mid$ rina similiter...»; f. $18^{\mathrm{r}}$ «Aristotelis Ethicorum liber primus | explicit. Incipit secundus feliciter» inc.: «Cum vero duplex sit virt | us...»; f. $26^{\mathrm{v}}$ «Ethicorum liber secundus explicit. | Incipit tertius feliciter.» f. $27^{\mathrm{r}}$ inc.: «Igitur cum virtus circa | affectus...»; f. 40" «Explicit tertius incipit quartus.» inc.: «De liberalitate deinceps | dicamus. Ea...»; f.53v «Explicit liber quartus incipit quintus» inc.: «De iustitia et iniustitia | considerandum...»; f. $67^{\mathrm{r}}$ «Explicit liber quintus incipit sextus» inc.: «Quoniam prius diximus $\mid$ medium esse...»; f. $76^{\mathrm{r}}$ «Explicit liber sextus incipit septimus.» inc.: «Post hec dicendum est $\mid$ alio...»; f. $90^{\mathrm{r}}$ «Explicit liber septimus incipit octavus» inc.: «Post hec consequens e | rit de...»; f. $102^{\mathrm{r}}$ «Explicit liber octavus incipit nonus.» inc.: «In cunctis autem dissimi | lium specierum»; f. $113^{\mathrm{r}}$ «Explicit liber nonus incipit decimus. Feli:.» inc.: «Sequitur fortasse nunc ut $\mid$ de...»; f. $126^{\mathrm{r}}$ expl.: «...sit et quibus legibus et moribus».

Se aprecian rasuras marginales, que probablemente contenían notas o glosas, en: f. $5^{\mathrm{r}}(11.8-10)$; f. $20^{\mathrm{r}}$ (11. 12 y 23 , hacen referencia a «ad illa et circa illa» y «repudiandum que», respectivamente, corregidas por el autor en el cuerpo del texto); f. $26^{\mathrm{r}}$ $\left(1.6\right.$, hacía referencia a «vocem») f. $31^{\mathrm{r}}(1.3)$; f. $45^{\mathrm{v}}(1.14$, hacía referencia a una posible variante textual de «gradiens purpuratus»); f. 49 $(1.17$, posible referencia a la omisión de «utrunque», añadida en el margen por el autor); f. 50 ${ }^{\mathrm{r}}$ (1.25, en el margen inferior se lee «ras.»); f. $106^{v}(1.12$, probablemente hiciera referencia a «coopinatio» corregida también por el autor en el cuerpo del texto); f. $108^{\mathrm{v}}(1.16$, en referencia a «ceteri omnes», corregido también por el autor en el cuerpo del texto); f. $116^{\mathrm{v}}$; f. $123^{\mathrm{r}}$ (11. 18-25, una mano posterior añade «nota» en el espacio de la rasura).

Bibl.: GARUFI 1902, p.231 nº 14; KRISTELLER 1963-1992, vol. I, p.393; VITI 1992, p.303;

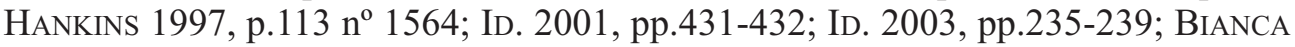
2002, pp.145, 149, tav. 7; BANDINI 2007, p.39 n. 19; FERRARO 2011, pp.75, 82.

\subsection{LA COPIA}

La escritura es una humanística redonda del primer cuarto del siglo XV y cumple con muchas de las características apuntadas por de la Mare (1977, p.91) para esta escritura en los primeros años de dicha centuria. Todo el códice parece haber sido co- 
piado por una misma mano, bastante uniforme aunque no del todo estable, con la misma tinta marrón y que se ajusta regularmente a la caja de escritura; también parecen de la misma mano los títulos en rojo, en todos la misma tinta (que coincide con el rojo de la decoración de las iniciales) y en mayúsculas con forma capital antigua (que coinciden con las del resto del texto). Apenas se aprecia el empleo de abreviaturas y en su mayoría se corresponden con los signos habituales: se indica el rasgo de nasalidad, el de ciertas desinencias como -us, -rum, -ibus, para -que, quam, vero, signo tironiano para et / -et / etiam; clara separación de letras; ' $\mathrm{d}$ ' con trazo recto vertical; ' $\mathrm{s}$ ' con trazo recto vertical alto; ausencia total de 's' redonda; ligadura 'ct' (inestable) y ' $\mathrm{st}^{6}$; restauración de la forma clásica nihil (sólo en una veintena de ocasiones aparece nichil) $)^{15}$, así como el diptongo ae. La restitución de ae es muy regular en casi todas las posiciones (interior de palabra y desinencias) y suele estar representado de forma separada y en menor medida con cedilla bajo la ' $\mathrm{e}$ '. Aunque se siente el esfuerzo del copista por esta restitución hay sin embargo lapsus, normalmente en el empleo de ciertos vocablos con sufijo prae-, como por ejemplo praeclarus (y derivados) que aparece un buen número de veces bajo la forma preclarus, praesens (y derivados) frente a presens; pero también en las desinencias y en el interior de palabra como quaero (y conjugación) que directamente se realiza en quero (dos veces), quaestio (y derivación; dos veces) frente a questio (dos veces y una con $e$ con cedilla), o sepe (una vez) por saepe (once veces). También se encuentran ejemplos contrarios, es decir, ultracorrecciones del tipo quaendam por quendam (una vez), praemor por premor (una vez), aepulum/-ae por epulum/-ae (de las siete veces que aparece sólo dos presenta la forma correcta), quaerela por querela (en las siete veces que aparece).

No se aprecia un interés, sin embargo, por restituir el diptongo oe ya que éste sólo se ha documentado la ultracorrección coeteris por cetereis (seis veces), el resto se resuelve en e: oeconomicum > economicum; moechus $>$ mechus; comoedus $>$ comedis; $c i$ tharoedo $>$ citharedo. En torno a este diptongo se ha comprobado cierta incertidumbre y así confunde coetus en caetus (las tres veces que aparece); poena en paena (sólo en dos ocasiones frente a las otras veinte que aparece ya sin diptongo pena). Y tampoco faltan ejemplos de ultracorrección: accoepit por accepit (las tres veces que aparece), accoepisse por accepisse (la única vez que aparece). Es acusada la inestabilidad de $y / i$ en palabras y nombres propios de origen griego: citharedi, gymnastica, schytarum, tyrannus, Polydamas pero también cytharedi, cytharam, misteriis, pyrate, tirannus, Olimpia, Policletum, Ciprigenam.

Una última característica sobresaliente del usus scribendi del copista tiene que ver con la pronunciación: se aprecia un intento de no emplear la palatalización $t i>c i, \mathrm{y}$ ejemplo de ello es amicitia: la palatalización amicicia es frecuente en los primeros libros y al principio del libro VIII pero va progresivamente desapareciendo y se restituye amicitia. En el resto de los casos sólo se advierte palatalización en: gratia $>$ gracia, una vez; pretium (y derivados)> precium, en tres ocasiones; patior (y conjugación) > pacior, cuatro veces; mollities $>$ mollicies, una vez. Es más acusado, sin embargo, el caso contrario, es decir la ultracorrección $c i>t i$ (sufficiat $>$ suffitiat; iudicium>iuditium;

${ }^{15}$ En la Praemissio aparece dos veces mihi (f. $1^{\mathrm{v}}, 1.6 ;$ f. $2^{\mathrm{r}}, 1.1$ ), ninguna en el texto de la Ética. 
mendacium $>$ mendatium; etc.) que se comprueba especialmente en el uso mayoritario de speties por species (aunque en el libro $\mathrm{X}$ se hace casi siempre un uso correcto) y benefitium por beneficium.

Hay intervenciones por parte del copista que se situarían en el momento mismo de la copia ya que, aparte de que se deban a la misma mano, se advierte el empleo de la misma tinta. Esas intervenciones, en las que se subsanan los errores de los que se percata el copista, se diferencian a distintos niveles:

- gráfico (atribuibles a olvido o por despiste), que se ejecutan de varios modos: marcando la letra con un punto debajo como en f. $9^{\mathrm{v}}(1.25)$ y f. $11^{\mathrm{v}}(1.16)$ iis ex hiis; marcando con puntos debajo y en rasura, y así en $\mathrm{f} .108^{\mathrm{r}}$ (1.7) honestatem ex hoehonestatem; o marcando con puntos debajo o con un signo de ' $\mathrm{V}$ ' invertida, o cuña, y añadiendo letras voladas como en f. $6^{\mathrm{v}}(1.20) \boldsymbol{h i s}$ ex is, f. $8^{\mathrm{r}}$ (1.23) substantia ex sustantia, f. 98 ${ }^{\mathrm{v}}(1.7)$ nut $[\mathrm{r}]$ iendique ex nutendique, f. $101^{\mathrm{v}}$ (1.14) $p$ [r]imo ex prmo, f. $107^{\mathrm{r}}$ (1.17) soliciti ex solici, f. 118v (1.3) prop $[\mathrm{r}]$ ia ex propa; en rasura corrige f. $122 \mathrm{v} 1.12$ cetera ex coetera y 1.24 ceterumque ex coeterumque.

- morfológico (atribuibles a errores gramaticales de los que se percata o de significado): f. $11^{\mathrm{v}}$ (1.18) dissonat ex dissonant (marca con un punto debajo de la $n$ y la tacha con un trazo oblicuo); f. $13^{\mathrm{r}}(1.4)$ amici ex amice (marca con un punto debajo de la $e$ y añade $i$ volada); f. 42v (1.19) recte ex rectum (marca con puntos debajo de $-u m$ y añade una $e$ volada); f. $63^{v}$ (1.17) omnis ex omnes (marca con un punto debajo de la $e$ y añade $i$ volada); f. $85^{\text {r }}$ (1.7) cervicis ex cervices (marca con un punto debajo de la $e$ y añade $i$ volada); f. $115^{\mathrm{v}}(1.2)$ effectiones ex affectiones (marca con un punto debajo de la $a$, la tacha y añade una $e$ volada); f. $115^{\mathrm{v}}$ (1.3) nullius ex nulllus (marca con puntos debajo del segundo brazo de la $u$ y debajo de $s$ para leer así nulli- y añade en volada el signo para $-u s) ;$ f. $118^{\mathrm{v}}(1.23)$ aliis ex alios (marca con un punto debajo de la $o$ y añade una $i$ volada); f. $119^{\mathrm{r}}(1.22)$ fiet ex fit (marca con un signo en forma de cuña y añade una $e$ volada); f. $113^{\mathrm{r}}$ pueros ex pueris (marca con un punto debajo de $i \mathrm{y}$ añade $o$ volada).

- omisiones que enmienda: f. $4^{v}$ (1.3) et delectationem non oportere / (1.15) expetere autem nanquam dicat (añade en margen con un punto, trazo oblicuo y un signo en forma de cuña) / (1.19-20) inepta (añade en margen con dos puntos separados por una línea vertical y un signo en forma de cuña); f. $28^{r}$ (1.22) errorem (añade en margen con un signo de tres puntos en forma de triángulo); f. $29^{r}$ (1.2) aliud (añade en margen con un signo de tres puntos en forma de triángulo); f. $63^{\mathrm{v}}(1.20)$ i [n] (añade en letra volada con signo de nasalidad) ; 119v (1.5) est (añade sobre la línea).

El proceder del copista en este tipo de correcciones parece constante y se diferencia del resto de las intervenciones que aparecen en el manuscrito. Como veremos más adelante el códice sufrió otra revisión posterior, esta vez por parte del autor, en la que además de intervenir en los errores de copia no subsanados por el copista, se ofrecen interesantes variantes relativas a la propia labor de traducción. Además, 
quizá sea esta la característica más sobresaliente, se corrigen, casi siempre en rasura, la mayoría de los diptongos ae, reflejo de las preferencias gráficas de Leonardo Bruni.

Volviendo a la labor del copista, se podría determinar que la ejecución es muy similar a la del escriba anónimo que copió varios códices que pertenecieron a Antonio Corbinelli ${ }^{16}$. Tal copista anónimo parece que trabajó bajo la guía de Niccolò Niccoli (Ullman 1960, p.85; Ceccanti 1993-1996, p.13) e incluso se ha apuntado la posibilidad de que fuera copia del propio A. Corbinelli (Gentile 1992, p.64). Aunque como decimos la ejecución es muy similar, los códices de Corbinelli presentan una escritura todavía más uniforme que la de $\mathrm{M}$. Por otro lado, no hemos podido constatar que el códice de Monreale perteneciera también al humanista florentino ${ }^{17}$, por lo que habría que descartar la identificación antes apuntada.

Sin embargo, no hay que descartar que el manuscrito monrealense hubiera sido copiado también bajo la supervisión de Niccoli. De hecho hay ciertos rasgos en la escritura del códice de Monreale que coinciden con la 'formal hand' del humanista (de la Mare 1973, pp.52-55) ${ }^{18}$. La escritura de Niccoli tiende a una ligera inclinación incluso en su letra más formal, rasgo que no está presente sin embargo en el códice de Monreale. Hay, por el contrario, usos del copista que podrían estar en relación con este personaje o con la producción a él ligada: la 'í' se marca con acento largo (sobre todo en los casos de fluctuación 'ii' / 'u', pero no sólo), la 'Q' es muy parecida a la de Niccoli con un trazo descendente bastante acusado, así como la ligadura 'ct' característica por el signo (en forma de sombrero) que se inicia en la ' $t$ ' $y$ desciende sobre la 'c'.

Por otra parte, si se acepta que Niccolò Niccoli fue el precursor o inventor junto con Poggio Bracciolini de la litttera antiqua ${ }^{19}$, y que éste último copió muchos códices alentado y bajo las indicaciones de su amigo Niccoli, no es extraño que los rasgos antes mencionados se puedan advertir también en la caligrafía de los seguidores de ambos e incluso en el segundo. Así, hemos podido constatar características de la escritura de Poggio Bracciolini o de la producción ligada a él en M: la 'g' minúscula (con el lóbulo inferior grande, aunque ligeramente más pequeño que el de los códices tempranos, cerrado y ladeado a la izquierda), la 'V' mayúscula (en la que el brazo izquierdo tiene un trazo hacia el exterior alargado a veces) y la forma del signo tironiano son 'pog-

\footnotetext{
${ }^{16}$ Códices laurencianos Plut. 51.4, Conv. soppr. 111 y Conv.soppr. 131, procedentes de la Badia florentina incluidos entre los libros que donó Antonio Corbinelli (Rollo 2004; Pomaro 1986, pp.239 y n. 10; de la Mare 1977, pp.96-97, fig. 3; Ulman 1960, p.81; Blum 1951, p.90, ad indicem).

${ }^{17}$ De entre los códices que pertencieron a A. Corbinelli, según el inventario cuatrocentesco que ofrece Blum (1951, pp.103) hay dos códices perdidos antes de $1504\left(\mathrm{n}^{\mathrm{os}} 45\right.$ y 46) y que contenían obras de Aristóteles, pero se trata ciertamente de códices griegos.

${ }^{18}$ Para las características de la escritura de Niccoli cf. Ullman (1960, pp.59-77); de la Mare (1973, pp.4461).

${ }^{19}$ Sobre las características de esta renovación gráfica, empleada por los humanistas fundamentalmente para la copia de autores clásicos, existe una amplia bibliografía por lo que remitimos a los estudios de Ullman (1960); de la Mare (1973; 1977); de la Mare- Thomson (1973); Casamassima (1974), Billanovich (1981), De Robertis $(1998 ; 2006 ; 2010)$ y a la bibliografía citada en ellos.
} 
gianos', al menos en su realización más tardía, y recuerda a la de los códices de la B. Vaticana Vat. lat. 3245 (ca. 1410-1414) y al laurenciano Plut. 48.22 (a. 1425), también a los códices atribuidos a un copista francés que trabajó a su servicio durante la década de $1420^{20}$. Podría ponerse también en relación con P. Bracciolini la inestabilidad ante formas coepit por cepit, sepe por saepe, el empleo constante de nihil, del signo de interrogación caracterizado por dos bucles inclinados hacia la izquierda (de la Mare 1973, p.70), la división anómala de palabras al final de línea (cf. Ullman 1960, p.27 y passim), por ejemplo, f. $5^{\mathrm{r}} 11.19-20$ doctr | ina, f. $21^{\mathrm{r}} 1.4$ qu | odamodo, $120^{\mathrm{r}} 1$. 11 neque $\mid a t$; o la ausencia de indicación con guión cuando hay separación de palabra también al final de línea (Billanovich 1981, p.133).

La ausencia de firma, marcas de posesión u otras que indiquen la procedencia del códice de Monreale hace difícil su identificación y sólo un paleógrafo podría determinar la autoría de la copia de Monreale. Aunque se han conservado copias autógrafas de Niccoli, de Bracciolini y de alguno de sus escribas, en muchos otros casos sólo se puede hablar de atribuciones ${ }^{21}$. Entre las atribuciones a P. Bracciolini encontramos, por ejemplo, el Plut. 48.34: datado entre 1403 y 1408, contiene las Filípicas de Cicerón y ha sido considerado como uno de los primeros ejemplos de imitación de la letra 'antigua' (de la Mare 1973, p.70-71, $76 \mathrm{n}^{\circ}$ 5) en lo que se ha llamado la 'restauración de la littera antiquae formae'. De un primer cotejo entre los laurencianos Plut. 48.34 y Plut. 48.22, autógrafo de Bracciolini (a. 1425) ${ }^{22}$ con el códice de Monreale se puede determinar que tanto la letra como las iniciales 'a bianchi girari' (de las que nos ocuparemos más adelante) son muy similares entre sí, y se podría establecer, dadas las características apuntadas más arriba, que $\mathrm{M}$ fuera un estadio intermedio entre la letra que presentan los códices 'poggianos' tempranos y la de los situados en torno a 1425.

De hecho ni en M ni en el Plut. 48.22 encontramos ya la 'A' uncial ni el punto sobre la ' $y$ ' característicos de la primera época de Bracciolini, el lóbulo de la 'g' minúscula es más pequeño y la ligadura de 'ct' no es tan exagerada como en el Plut. 48.34. En el Plut. 48.22, se aprecia ya una ligera diferencia entre ' $u$ ' $y$ ' $v$ ' (no apreciable en M), y es destacable la ausencia del diptongo ae, excepto en Caesar (cf. ULMAN 1960, p.34, 53). En fin, entre las características y el modus operandi de la escritura a la antiqua, Billanovich (1981, p.131) establece que tanto el pautado a punta seca como los títulos en mayúscula son característicos de las copias tardías de Bracciolini y de sus imitadores, por lo que M podría situarse perfectamente en el ámbito de dicho copista.

A estos datos codicológicos se pueden añadir otros de tipo documental que pueden ayudar a situar la copia de Monreale en el ambiente inmediato de estos dos per-

\footnotetext{
${ }^{20}$ Para este copista, la evolución de su escritura y los manuscritos a él atribuidos cf. Ullman (1960, pp.41, 44, 47-48); de la Mare (1973, pp.72-73); de la Mare - Thomson (1973); Billanovich (1981).

${ }^{21}$ De Robertis (2006, pp.133-134) ofrece un útil listado de los principales copistas florentinos en littera antiqua de la primera generación (1397-1425).

${ }^{22}$ Contiene Filipicas y Catilinarias de Cicerón, cf. Ulman 1960, p.34-36 y de la Mare 1973, pp.69-74, para las características (a veces divergentes de estos estudios) sobre la escritura de Bracciolini en este códice y en este período.
} 
sonajes. La traducción de la Ética se acabó en $1417^{23}$, pero en el otoño de ese año no estaba todavía 'corregida' como se lee en una carta de Bruni a Lorenzo de' Medici ${ }^{24}$ (Ep IV.11 [13]): Fines Bonorum a te expecto. Nam Aristotelis Ethica, quae traducere coeperam, nuper absolvi. Ea cum expolire nunc cupiam, Cicerone, ac Finibus illis opus est (cf. Mehus 1741, I, p.125). En una carta anterior (Ep. IV.10 [12]), con fecha de 26 de septiembre de 1417, dirigida a Niccolò Niccoli, Bruni pide a su amigo que se prepare para copiar nuevamente toda la Ética: Libros Ethicorum divinissimos ut ex integro rescribas, iam nunc te para (Mehus 1741, I, p.124). De esta información parece evidente que la obra había sido ya copiada parcialmente por Niccoli lo que le sitúa como candidato idóneo a ser, si no el autor, el supervisor del apógrafo originado a partir del ideógrafo íntegro recibido ${ }^{25}$.

De ser así, Bruni debió de hacer llegar un ideógrafo de la Ética a Niccoli antes de 1419 , pues sabemos que en ese año surgió una enemistad entre ambos personajes ${ }^{26}$. La Ética se acompaña de dos prefacios (Baron 1928, pp.75-76): una praemissio en la que Bruni justificaba su traducción y una praefatio dedicatoria a Martín V. Su publicación con la praefatio no pudo ser anterior al 11 de noviembre de 1417, fecha en la que Martín V fue coronado Papa (Gualdo Rosa 1981, p.88 n.35) pero la decisión de Bruni de dedicarle la obra parece que fue posterior. Según Hankins (2003, p.194, n. 2) la presentación de la dedicatoria ante el pontífice se habría producido entre febrero de 1419 y septiembre de 1420, período en el que Bruni trabajó a su servicio durante la estancia de Martín V en Florencia. Sólo dos manuscritos que pueden datarse en fecha temprana, el Plut. 79.7 y el de Monreale, presentan el texto de la Ética acompañado únicamente por la Praemissio, lo que podría situarlos en el entorno de la producción de Niccoli. La copia de Monreale, a juzgar por los rasgos caligráficos y por la decoración, que veremos más adelante, se trata de un ejemplar de lujo (por lo que la versión ya estaba acabada) y parece anterior a la copia que hizo Giovanni Aretino para Cosimo de' Medici conservada en el códice Plut. 79.7, que puede datarse entre $1418-1420^{27}$.

La Ética debió empezar a circular en su versión 'pulida', tal y como anunciaba el propio Bruni, a finales de 1417 o en la primera mitad de $1418^{28}$. Todo parece indicar

\footnotetext{
${ }^{23}$ Según Gualdo Rosa (1980, p.88 n.35), L. Bruni acabó la traducción entre 1416 y marzo de 1417, pero el trabajo de revisión y corrección se retrasó hasta la segunda mitad de 1417.

${ }^{24}$ Según supone Gualdo Rosa (1980, p.88 n.37), Leonardus Aretinus s. d. p.Laurentio. En la edición de Mehus (1741, p.125) se lee Leonardus Laurentio $S$.

${ }^{25}$ Como se ha señalado (cf. supra nota 5) se sabe que Bruni dictaba sus traducciones a un amanuense, normalmente tenía un copista a su servicio (Venier 2011, p.172), por lo que hay que pensar que Niccoli pudo recibir un ideógrafo a partir del cual se realizó un apógrafo del que derivarían las primeras copias.

${ }^{26}$ Cf. la Ep.IV.22 (V.4) de Bruni a Bracciollini en la que explica los motivos de esa enemistad que duró hasta 1426 (Mehus 1741, II, pp.17-25).

${ }^{27}$ Probablemente es posterior a 1418 ya que no aparece en el inventario de ese año de la biblioteca de Cosimo de' Medici (Pintor 1902; Ullman 1960, p.94 nº 11). Para la datación cf. Ceccanti (1993/1996, p.13) y Scarcia (1981, p.137).

${ }^{28}$ La Ep.IV.15 (X.26) de Bruni a Giannicola Salerno, de la segunda mitad de 1418, constituye la respuesta de Bruni a la primera crítica oficial de su traducción. La traducción de la Ética generó numerosas polémicas a lo largo del s. XV (Garin 1993, p.11; Hankins 20003, pp.193-239) pero quizá la que más interés ha suscitado
} 
pues que la ejecución de la copia de Monreale se situaría también en este período. Esto no quiere decir que el autor no volviese sobre su versión: las correcciones y anotaciones autógrafas posteriores que contiene el códice indican de una parte la preocupación del autor por la corrección de las copias que circulaban, y por otra una revisión de su propia labor de traducción.

Por su parte, Poggio Bracciolini entre 1414 y 1418 se encontraba en Constanza ${ }^{29}$ y de allí pasó a Inglaterra al servicio de Henry Beaufort, arzobispo de Winchester, hasta la segunda mitad de 1422. Parece extraño que no haya noticias de que Bracciolini hubiera hecho una copia de esta versión de Bruni ${ }^{30}$, pues dada la amistad y colaboración que unía a ambos personajes lo lógico hubiera sido que en alguna ocasión se mencionara en el caso de haberse llevado a $\mathrm{cabo}^{31}$. De la correspondencia mantenida entre Niccoli, Poggio y Bruni sabemos del intercambio de libros entre estos tres personajes, sin embargo, en cuanto a la Ética y a Bracciolini se refiere sólo tenemos noticia de una nota de posesión de éste en el manuscrito laurenciano Plut. 79.10 ${ }^{32}$ de 1427.

\subsection{LA DECORACIÓN}

Una de las características que llama la atención del códice de Monreale es que la decoración 'a bianchi girari'33 de las iniciales es muy temprana y muy similar a la que ofrecen los laurencianos Plut. 37.2 (a. 1404), Conv. soppr. 111 (a. 1405) o Conv. soppr. 131 (a. 1406) pero no son de la misma factura ${ }^{34}$. Sin embargo, la primera inicial miniada del códice monrealense, f. $1^{\mathrm{r}}$, es casi idéntica a la del Laur. Plut. 48.34, f. $1^{\mathrm{r}}$. (Ceccanti 1993/1996, fig. 2) ${ }^{35}$. El resto de las iniciales de M son de la misma factura

entre los investigadores fue la mantenida por el propio Leonardo Bruni y Pier Candido Decembrio con Alfonso de Cartagena entre 1436 y 1439, conocida como Controversia Alphonsiana (Birkenmajer 1922; González Rolán et alii 2000; Morrás 2002).

${ }^{29}$ Durante el concilio que allí se celebró (1414-1417) y en el que fue elegido papa Martín V.

${ }^{30}$ Sabemos que en Inglaterra estudió los comentarios de Santo Tomás a la Fisica de Aristóteles (Ullman 1960 , p.50 $\mathrm{n}^{\mathrm{o}}$ 7) como resulta de las notas en el ms. BNCF, Conv. Sopp.J.V.42, y de la Ep.5 (a. 1420) y Ep.8 (a. 1421) dirigidas a N. Niccoli (editadas por Hart 1984a, pp.15-15, 22-29).

${ }^{31}$ Cf., por ejemplo, la correspondencia entre ambos personajes (Mehus 1741; Gualdo Rosa 1981; Hart 1984b).

32 En el f.80': «Finit bonis avibus. Anno DNI 1427 mense sept. die XV hora none per [ras.]. Liber Poggii. Secretarii Apostolici.». Contiene la versión de la Ética (Hankins 1997, p.43 nº 550).

33 Sigue siendo imprescindible para una primera aproximación el estudio de Pächt (1957). Cf. también Ceccanti (1993/1996).

34 Para la descripción de las iniciales de estos códices vid. De Robertis (2006, p.116 n. 17). Las iniciales del Plut. 37.2 son muy parecidas pero parecen menos evolucionadas que las de Monreale. Sin embargo, las iniciales del Conv. soppr. 111 y 131, se diferencian porque (sólo la letra) en vez ser amarillas están esmaltadas en oro, lo que supone un estadio intermedio entre las anteriores y las que presentan códices posteriores datables en la segunda decena del s.XV (los Plut. 48.10 y 79.8, por ejemplo).

35 El códice Laur. Plut. 48.34, como todos los Pluteos, se puede consultar libremente online en la página web de la Biblioteca Medicea Laurenziana: http://www.bml.firenze.sbn.it/ (última consulta 29/04/2012). Hemos obtenido sin embargo la autorización de dicha biblioteca para la publicación de la inicial del manuscrito Plut. 48.34 f. $1^{\mathrm{r}}$. 


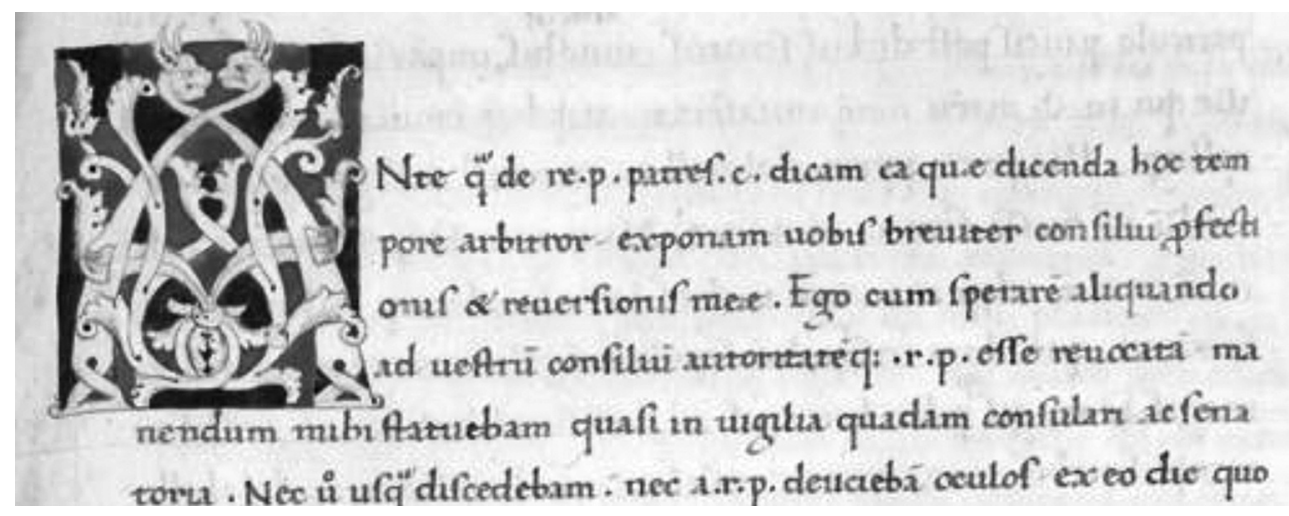

Fig. 3. Biblioteca Medicea Laurenziana, Plut. 48.34 f. $1^{\mathrm{r}}$.

que las que aparecen en el interior del Plut. $48.34^{36}$ y también las que se encuentran en los laurencianos Strozzi 96, Plut. 48.7, Plut. 67.11, Plut. 48.22 y Plut. 51.31, todos ellos autógrafos de Bracciolini ${ }^{37}$.

La inicial que aparece en el laurenciano Plut. 48.34, f. $1^{\mathrm{r}}$, ha sido definida por Ceccanti (1993/1996, p.12):

Il Laur. Pluteo 48.34 (...) mostra come ascendenti diretti i codici in stile geometrico o vegetale primitivo e di transizione, dove la lettera è formata da nastri che potremo definire a "full-shaft" con intrecci alla base e un motivo zoomorfo all'apice realizzato con una qualche abilità.

La autora apunta, en un primer momento, la posibilidad de que la miniatura sea obra del propio Bracciolini. Esta hipótesis será recogida también por Ciccuto (2007) para los códices autógrafos del humanista antes señalados, copiados bajo el estímulo y mecenazgo de Niccoli, considerado el inspirador del nuevo estilo decorativo. Se basa para ello en la correspondencia entre texto-imagen que se advierte en dichos manuscritos, es decir entre «fatti paleografici (la scrittura riformata) e fatti artistici (i "bianchi girari")» (Ciccuto 2007, p.58), y sostiene que, fruto del interés paleográfico y artístico y de la estrecha colaboración Niccoli-Bracciolini, estos códices son el punto de partida de la posterior imitación de los modelos románicos.

Sin embargo Bernasconi (et al. 1993, p.83) considera que las iniciales del Plut. 48.34 (y las de otros autógrafos de P. Bracciolini: Strozzi 96, Plut. 48.27, Plut. 67.11) son obra de iluminadores profesionales que trabajaban seguramente bajo las indica-

\footnotetext{
${ }^{36}$ Excepto la que aparece en el $\mathrm{f} .41^{\mathrm{v}}$ pues se añadió un cuaderno con la parte que faltaba de la obra, atribuido éste a Ambrogio Traversari (Bernasconi 2000, pp.56-57).

${ }^{37}$ El primero datado entre 1402-1403, los dos siguientes entre 1403-1408 y los dos últimos en 1425. Bernasconi et al. (1993, p.82) señala que son de la misma factura pero que los dos últimos «se distinguent par la présence d'initales bleues avec rinceaux dessinés à l'ence rose et rouge».
} 


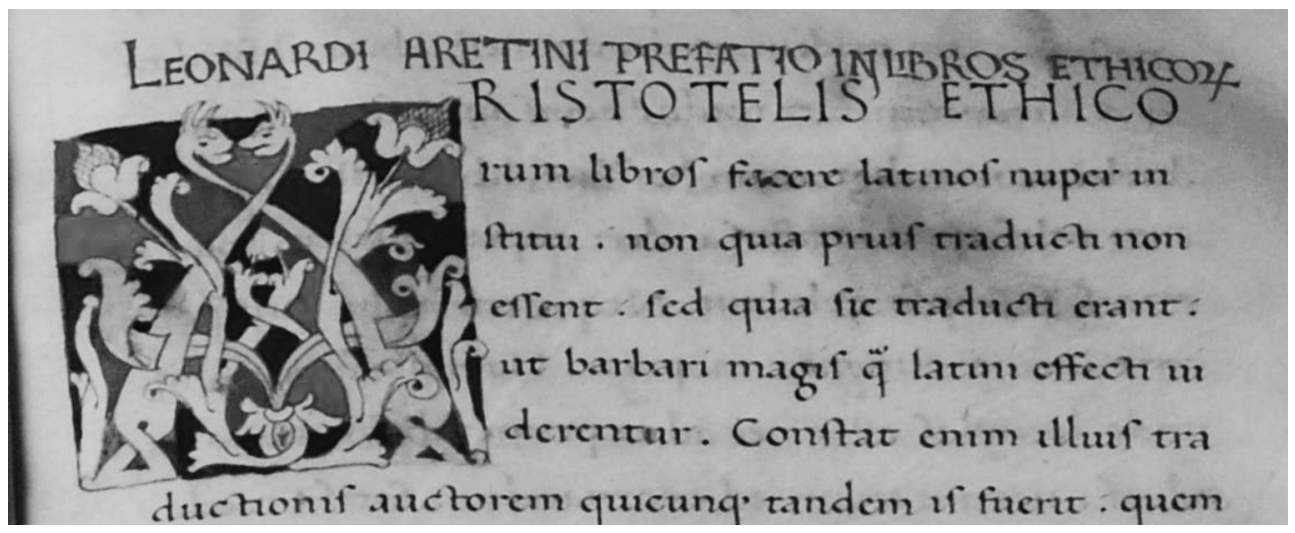

Fig. 4. Monreale, Biblioteca Comunale Santa Maria la Nuova, XXV F 10, f. $1^{\mathrm{r}}$.

ciones de Niccoli ${ }^{38}$. Para esta tarea de iluminación Ceccanti (1993/1996, p.16) señala el convento de Santa Maria degli Angeli, en Florencia, como ambiente cultural dirigido por Ambrogio Traversari y en el que sitúa un scriptorium que bien podría ser el lugar de elaboración del códice que nos ocupa.

Por su parte, Ferraro (2011, p.82) se refiere en los siguientes términos a las iniciales del códice de Monreale:

(...) iniziali a bianchi girari, poste ad inizio di ogni libro, in cui l'intreccio stilizzato è simile a nastri con foglie, pigne e teste di animali.

En realidad, la inestabilidad en el encuadramiento en relación con el espacio para los títulos y el texto de la primera inicial en el códice de Monreale invita a pensar en un artífice no profesional. Su identificación con la del Plut. 48.34, así como la factura particular de los colores del resto de las iniciales interiores de los códices 'poggianos" confirman la ejecución de un mismo iluminador o bodega que operaba en Florencia entre 1402 y 1425 .

\subsection{CORRECCIONES AUTÓGRAFAS DE LEONARDO BRUNI}

Además de todas estas características extraordinarias que presenta el códice de Monreale desde el punto de vista codicológico, hay que añadir su enorme valor desde el punto de vista textual pues las correcciones autógrafas que presenta le hacen adquirir la categoría de 'arquetipo', según la acepción de Perosa (2000, p.14), ya que estará a la cabeza de una parte limitada de la tradición manuscrita, generando a su vez

\footnotetext{
${ }^{38}$ Se sabe que Bracciolini y Bruni mandaban a Niccoli sus códices para que fueran decorados (de la Mare 1973, p.74; Bernasconi et alii 1993, p.83 n. 64; Bernasconi 2000, p.56).
} 
una nueva familia ${ }^{39}$. El punto en el que se encuentran nuestras investigaciones sobre la tradición manuscrita de la versión bruniana de la Ética no nos permite trazar aún un esquema completo de la relación entre los testimonios de la misma, por lo que nos limitaremos a señalar en este lugar únicamente los tipos de intervención del autor en este códice.

Se pueden establecer varias fases en la elaboración del manuscrito de Monreale. Una primera correspondería a la copia y a una primera corrección de la misma como hemos apuntado más arriba, en la que el propio copista se percata y corrige los errores del proceso de copia. El texto sufrió una segunda revisión por parte del propio autor, tal como se expresa en la subscriptio, cuando el texto ya estaba en circulación: como se ha visto se trata de un códice de lujo, dadas sus características codicológicas, y además muchas de las lecturas que se corrigen en $\mathrm{M}$ no son subsanadas por otros códices, lo que indica que las correcciones se hicieron con posterioridad a una primera puesta en circulación de la versión. No sabemos las causas de la corrección de este códice, pero no parece que estuviera destinado a ser modelo de ningún otro, sino simplemente que lo corrigió, tal vez para enviárselo a algún conocido, como era práctica habitual de Bruni (vid. supra nota 9).

En esta segunda revisión o fase se aprecian dos tipos de intervenciones: por un lado las correcciones propiamente dichas a la labor del copista y que afectan a los distintos niveles de escritura: gráfico, morfológico y sintáctico (en el que se incluirían también la subsanación de las omisiones del copista); por otro lado, hay intervenciones que precisan distintos aspectos de la traducción. Esto es, Bruni interviene en el primer caso como 'corrector' (o 'copista'), colacionando probablemente a partir de su propia copia de autor, y en el segundo caso como 'autor', modificando el texto que ofrece la copia que está corrigiendo ${ }^{40}$, bien por razones estilísticas, esto es en cuanto al texto latino se refiere, bien ofreciendo variantes de la propia traducción, fruto de la comparación de distintos códices griegos ${ }^{41}$.

El modo de proceder es diferente al de la primera corrección del copista: la tinta es distinta a la empleada para la copia (es más oscura), en esta ocasión prevalece la corrección in rasura o in margine con la inclusión de $\operatorname{signos}^{42}$, que marcan el lugar preciso en el que se deben incluir las correcciones, y se indica también el cambio de orden en la frase mediante dos trazos oblicuos (como unas comillas rectas) sobre los elementos a alterar (por ejemplo f. 16 $16^{\mathrm{v}}$. 20. operiosus forsan ex forsan operiosus).

\footnotetext{
${ }^{39}$ Para Perosa (2000, pp.13-14) el material manuscrito de época humanística que nos llega es, por lo general, contemporáneo al autor y a menudo ha sufrido cambios y nuevas variantes. Considera que el verdadero arquetipo es el autógrafo y a menudo es un arquetipo en movimiento que se va transformando y modificando en el tiempo, y normalmente no se puede hablar de un único arquetipo sino de varios.

${ }^{40}$ Cf. Rizzo (1984², p.243 y ss.) para los distintos tipos de corrección después de la copia.

${ }^{41}$ Cf. Hankins (2003, p.237): «What such correctio and annotations may indicate is Bruni's continuing awareness of the gaps and deficiencies in his Greek manuscripts and an attempt to remedy them after the initial circulation of his Latin version».

${ }^{42}$ En general se emplean dos puntos, dos puntos debajo de un trazo horizontal y una cuña, un punto y un trazo oblicuo, tres puntos en forma de triángulo, trazo vertical, oblicuo o en forma de aspa para tachar letras y subrayado para indicar lo que debe eliminarse.
} 
En cuanto a las intervenciones gráficas lo más destacable es la voluntad de Leonardo Bruni en la restitución de la grafía e para el diptongo $a e^{43}$ : la mayoría de los diptongos ae se rasuran y en muchas ocasiones se sobrescribe $e$ (coincidiendo con la mano y tinta de las intervenciones del autor); otros fueron marcados para indicar que debían corregirse (con un punto debajo de la $a$ y tachada) pero no llegó a realizarse dicha corrección; muy pocos pasaron desapercibidos y no fueron corregidos ni marcados. No sucedió lo mismo con nihil pues no se corrigió ninguno en nichil a pesar de la preferencia de Bruni por esta forma (cf. Baron 1968, pp.219-223). Otras intervenciones a lo largo del códice son las relativas al fenómeno -ti->-ci-, y así corrige (f. $92^{\mathrm{r}}$ ) hospitalitiam ex hospitatieiam, (f. $121^{\mathrm{r}}$ ) otiemur ex ociemur, (f. $121^{\mathrm{r}}$ ) otium ex ocium, o a la ultracorrección -ci->-ti-, como (f. 69 ${ }^{\mathrm{r}}$ ) iuditium corregido en iudicium. Por último, prefiere quidpiam a quicpiam (f. $72^{\mathrm{r}}$ ) y quidquam a quicquam (f. $91^{\mathrm{r}}$ ).

Además de estas intervenciones sobre la copia, las anotaciones marginales (bien sean correcciones, subsanaciones por omisión previa, bien sean notas a la traducción) muestran el usus scribendi del autor y así se observa exclusivamente el empleo de $e$ por ae, el de nichil (ff. 17v, $70^{\mathrm{v}}$ y $116^{\mathrm{v}}$ ) frente al nihil mayoritario del copista, o la preferencia de - $m n$ - frente a la frecuente (pero inestable) -mpn- del copista, y así por ejemplo, en el f. $58^{r}$ (1.3) se lee: ille passus est, si hic damnum dedit. Sin embargo hay muchos usos que Bruni no ha corregido o señalado (itaque nescio satis emendatus sit) tal vez por la inestabilidad que presenta la copia y por la rapidez con la que declara haber corregido.

En verdad son muy numerosas las correcciones de Bruni a lo largo de todo el códice (sed emendatus tamen in plurimis) por lo que no podemos dar cuenta aquí de todas las modificaciones a nivel morfológico y sintáctico. Sin embargo hay que destacar que efectivamente la lectura de Bruni fue rápida (sed cursim) y en alguna ocasión también la corrección. Así, por ejemplo, en: (f. $23^{\mathrm{r}}$ 11.8-9) Et ob hoc igitur vitii est excessus et defectus. Virtutis autem medii corrige medii ex medium (lectura que comparten el resto de los manuscritos que hemos podido consultar). Dado el contexto sintáctico precedente y el correspondiente texto griego ${ }^{44}$ : (Arist., EN 1106b33-34) кaì

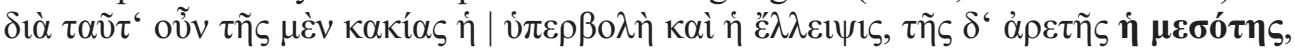
creemos que medium es la forma más adecuada y que la corrección en medii se trataría de un lapsus calami por un hipotético plural media, debido tal vez a razones estilísticas pero que no se debería aceptar de cara a la edición del texto.

\footnotetext{
43 Dichas correcciones como apunta Hankins (2003, p.235) están de acuerdo con las preferencias de Bruni y se oponen a la restauración de las formas clásicas iniciada por Niccolò Niccoli (Baron 1968, pp.219-223; Gualdo Rosa 1980, p.XII).

44 Seguimos la edición oxoniense de Bywater (1894 [1988]) aunque como demostraban Gauthier- Jolif (1970², "introduction") no hay hasta el momento ninguna edición crítica satisfactoria del texto griego. No hemos podido determinar de momento el modelo seguido por Bruni para su traducción. Hankins (2003, p.236 n.5) señala entre los posibles el veneciano Marcianus graecus 213, con el que hemos constatado además una omisión: (Arist.,

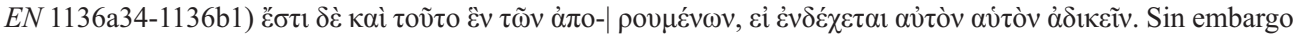
dicho códice llegó a poder del cardenal Besarión entorno a 1460, por lo que es improbable que Bruni tuviera acceso a él (Harlfinger 1971 [ ] p.465. De los ejemplos propuestos a continuación no se ha podido constatar ninguna variante imputable al texto griego y es por ello por lo que las hemos considerado «estilísticas».
} 
También a razones estilísticas pueden deberse las siguientes intervenciones: (f. $7^{\vee}$ 11.5-7) Qui non ipse videt neque credit recta monenti, hic nulla in parte utilis est, donde non ha sustituido un inicial nec (lectura que comparten la mayoría de los códices colacionados, sólo uno de ellos presenta non). La secuencia nec...neque es frecuente en el texto y también en el uso del autor, y además se adecua más con la sin-

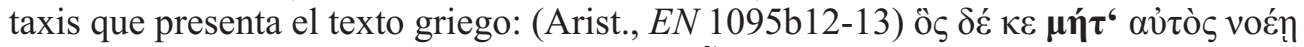

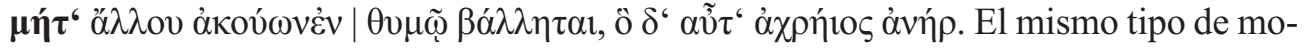
dificación lo encontramos también en: (f. 70 11.4-5) Hunc non fossorem dii fecerant, non aratorem, nec aliud quiddam sapientem, sustituyendo los iniciales neque y nec (lectura unánime de los manuscritos colacionados) y alejándose del modelo griego:

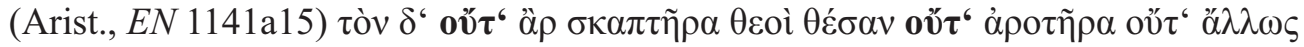
$\tau \imath$ бoழóv. En ambos casos se trata de citas del texto aristotélico, la primera referida a Hesiodo (Op.293-296), la segunda a (Ps.) Homero (Margites) por lo que creemos que la intervención ha sido voluntaria y meditada, queriendo reflejar en cierto modo una distancia entre su discurso y el de las citas.

Un último ejemplo de este tipo de modificaciones por posibles razones estilísticas hechas por Bruni en el códice de Monreale sería: (f. 73 1.1) At ignoscere sentire est recte iudicando circa equitatem. Recte autem vere. El texto griego dice: (Arist., EN

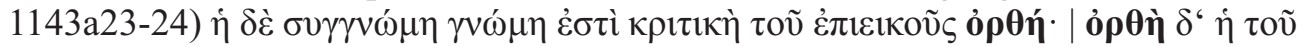
$\dot{\boldsymbol{\alpha}} \lambda \eta \boldsymbol{\theta} \boldsymbol{0} \mathbf{v} \varsigma$. La primera lectura de Monreale es recta autem veri (y también la de otros dos manuscritos colacionados), que es la lección que se acerca más a la sintaxis griega («recta en cuanto a lo verdadero»). El resto de los manuscritos sin embargo leen recte autem veri y por último Bruni corrige en Monreale recte autem vere. En realidad, Bruni cambia en su traducción la estructura sintáctica del texto griego, e incluso

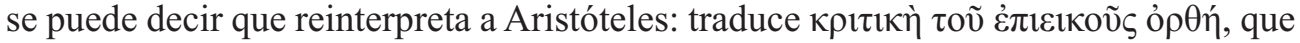
en el texto griego sería el atributo de una oración copulativa con sujeto $\dot{\eta} \delta \dot{\varepsilon} \sigma u \gamma \gamma v \omega ́ \mu \eta$ $\gamma v \omega ́ \mu \eta$, por el gerundio recte iudicando circa equitatem posiblemente por haber traducido el nominativo griego $\dot{\eta} \delta \dot{\varepsilon} \sigma v \gamma \gamma v \omega ́ \mu \eta \gamma \nu \omega ́ \mu \eta$ por un infinitivo (ignoscere), seguramente para dar continuidad a la traducción de la frase anterior (et aequum esse circa quaedam ignoscere $)^{45}$. Por otra parte, no sería tampoco extraño que Bruni hubiera constatado una variante $\dot{\alpha} \lambda \eta \theta \tilde{\omega} \varsigma$ en lugar de $\dot{\alpha} \lambda \eta \theta$ oṽ, , aunque, de ser así, lo hubiera indicado probablemente al margen, lo que nos lleva a pensar que este ejemplo podría tratarse de una conjetura del propio Bruni ${ }^{46}$.

En este sentido, y en cuanto a las variantes o matices de la traducción se refiere debidas al cotejo con otros códices griegos, se encuentran las anotaciones o dobles traducciones ofrecidas por el autor en el margen del manuscrito monrealense ${ }^{47}$, en lo que podríamos denominar un ejemplo de 'traducción crítica' por parte de Bruni, y de

\footnotetext{
45 Agradezco la precisa observación sobre este pasaje al Profesor Antonio Espigares.

${ }^{46}$ Que coincidiría precisamente con la que propone Trendelenburg $(1867$, p.432) y señalada en aparato en la edición del texto griego de la Ética de Susemihl (1882, p.138).

${ }^{47}$ Estas anotaciones se repiten en muchos otros códices. Hankins señala únicamente el Ricc. 114 y el Conv. Soppr. C7 2677 ¿entendiendo que las postillas que recogen ambos podrían ser también autógrafas? En cualquier caso muchos códices repiten dichas anotaciones: algunos interpolándolas en el propio texto pero la mayoría reproduciéndolas también en el margen, por lo que se sintieron claramente como variantes a la traducción.
} 
las que ya Hankins (2003, p.236) señaló el ejemplo del f. 56 $6^{\mathrm{v}}$ (Reperitur in quibusdam codicibus grecis isto modo: Sed quoniam iniquum et plus non est idem sed aliud, ut pars ad totum. Nam quod plus id omne iniquum, quod autem iniquum id non omne plus. Profecto et etc., cf. Fig. 2). Según este autor, la hipótesis más plausible, y con la que nosotros estamos de acuerdo, es que Bruni encontró distintas variantes una vez completada su traducción «adding them into the margin of this codex at a later time» y esas anotaciones se repiten de forma parecida, «but not identical», en otros códices, lo que indica una revisión y una intervención continua de Bruni en las copias puestas en circulación, y no sólo a partir de los códices griegos sino probablemente también de los comentaristas y de la otras versiones latinas anteriores, como la de Grosseteste $^{48}$.

Así, por ejemplo, en el f. $17^{\mathrm{v}}$ ante Quatenus autem non ulterius repugnat ofrece en el margen una alternativa a su propia traducción al. Qualiter autem aliud nichil differt que si bien parece adecuarse más al texto griego, (Arist., $E N 1102 b 25) \pi \tilde{\omega} \varsigma \delta$ ' ع̌ $\tau \varepsilon \rho o v$,

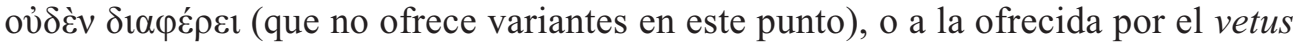
interpres: Qualiter autem alterum, nichil differt (cf. Gauthier 1973, p.394), no es acogida por ninguno de los códices colacionados ${ }^{49}$. Esa anotación se repite con variaciones en el Plut. 79.7 (f. $16^{\mathrm{v}}$ de una mano distinta a la del copista se lee alius textus: quomodo aut aliud nihil differt) y en el de la BNCF, Conv. Soppr. C7 2677 (f. $9^{\mathrm{r}}$ de la misma mano del copista se lee al. Qualiter autem aliud nihil differt rationis ec., y de una segunda mano Reperitur aliter in greco videlicet, quomodo autem aliud nihil differt).

También con la fórmula al. (alius textus; aliter, alias, alibi) se encuentra en el margen del f. $38^{v}$ una variante al término labiis : al. in saporibus, pero que no hemos constatado haya sido acogida por ningún otro manuscrito ${ }^{50}$. Por último, en el f. $45^{\mathrm{v}}$ hay una rasura en el margen sobre una anotación de la que son aún visibles tres puntos en forma de triángulo y lo que podría ser un al., que hacía referencia a gradiens

${ }^{48} \mathrm{El}$ autor conocía las versiones latinas anteriores realizadas a partir del griego: las parciales (s.XII), conocidas como Ethica vetus (libros II y III) y Ethica nova (libro I), atribuidas actualmente a Burgundio de Pisano (Brams 2009, pp.84-87), así como la versión completa de Roberto Grosseteste (ca. 1250), conocida como Liber Ethicorum. Esta versión integral se realizó a partir de la corrección de las dos versiones anteriores con nuevos códices griegos a lo que se añade la traducción completa de los otros siete libros junto con los comentarios de Eustracio (libros I y VI), Miguel de Éfeso (libros V, IX y X), Aspasio (libro VIII) y de un autor anónimo del s. XII (libro VI). Este Liber Ethicorum fue objeto de revisión entre 1250 y 1260 por parte de Guillermo de Moerbecke y fue el texto base para los comentarios de Tomás de Aquino (Marchesi 1904; Franceschini 1955; Gauthier 1970, 1973; Hankins 2003).

49 Varios códices, entre ellos el Plut. 79.11, copiado en Venecia por Giovanni Aretino entre 1422-1423 (U11man 1960, p.95; Scarcia 1981, p.137), los Escurialenses f.III.25 y p.III.22, Biblioteca Nacional de Madrid mss. 6927, 10104, 7687, o Biblioteca Ambrosiana mss. I 98 sup.y H 184 inf., incluyen interpolada en el propio cuerpo del texto dicha anotación incluso con variaciones como las que se señalan a continuación. Hay otros muchos códices, sobre todo de fecha tardía, que sustituyen el texto original por la variante, incluyendo en muchos casos variantes a su vez, por ejemplo: Bolonia Biblioteca Universitaria mss. 595 T (4) (non ante 1431), 1639, 2703 (a. 1457); Biblioteca Riccardiana mss. 135 (ca. 1456), 166 (ca. 1430-1441); Real Biblioteca de El Escorial, ms. f.II.3; Biblioteca Ambrosiana ms. L 47 sup.

${ }^{50}$ Tampoco parece que haya correspondencia con ningún mansucrito griego pues la lectura (Arist. $E N$, 1118a28) $\chi \cup \mu \tilde{\omega} v$, ofrece la variante $\chi \nu \lambda \tilde{\omega} v$ del Plut. 81.11 y del Parisiensis 1854, que no se corresponderían en ningún caso en sentido literal con labiis, y se adecuan sin embargo más con saporibus. 
purpuratus. El códice BNCF Conv. Soppr. C7 2677, f. $23^{v} 1.24$, lee efferens purpuram ${ }^{51}$ y de nuevo en el margen dos anotaciones: de la misma mano que copia el códice, al. gradiens purpuratus; de una segunda mano, al. inferens purpuram. El texto griego no

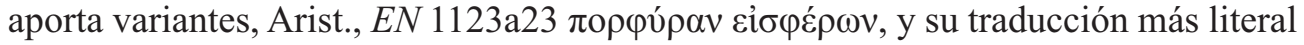
sería efectivamente inferens purpuram, como parece que advertía Argyropoulus en sus clases ${ }^{52}$. No sabemos qué se leía en la rasura del códice de Monreale, pero en cualquier caso debemos pensar que el texto que ha prevalecido, gradiens purpuram, refleja la voluntad del autor.

\subsection{LA CONSERVACIÓN EN MONREALE}

Sobre la llegada del códice a la biblioteca de la Abadía de Monreale, hoy sede de la Biblioteca Comunale Santa Maria la Nuova ${ }^{53}$, no tenemos ninguna noticia, y tampoco de su conservación en dicha biblioteca. De hecho el códice ha pasado desapercibido en los distintos catálogos e inventarios, antiguos y modernos, relativos a la biblioteca. ${ }^{54}$

Sabemos que perteneció al último Abad de Monreale (como todos los manuscritos medievales), Domenico Benedetto Gravina $(1807-1886)^{55}$, a la biblioteca privada de los Padres Benedictinos del monasterio de Monreale ${ }^{56}$. El convento de Santa Maria degli Angeli de Florencia (posible lugar de iluminación del códice) pertenecía también a la orden benedictina, pero no ha quedado registro de un posible intercambio de libros entre dichos monasterios.

\section{CONCLUSIONES}

El manuscrito XXV F 10 de la Biblioteca Comunale Santa Maria la Nuova de Monreale es un testimonio excepcional que recoge distintos aspectos característicos del primer humanismo italiano del Quattrocento. Desde el punto de vista cultural, aunque este aspecto no ha sido objeto de este estudio, contiene una de las obras fundamentales para la concepción y el desarrollo del humanismo cívico florentino: la versión bruniana de la Ética a Nicómaco, que fue acogida por universidades y círculos intelectuales de toda Europa como texto para la enseñanza de la filosofía práctica, además de generar una

\footnotetext{
51 Y también los de la Biblioteca Ambrosiana mss. R.79 sup. (a. 1425) y B 166 suss.

${ }^{52}$ Como señala Hankins (2003, p.229), en el códice 2703 de la Biblioteca Univesitaria de Bolonia (vid. también nota 49), anotado por Benedetto Colucci cuando asistía a las clases de Argyropoulus, encontramos la siguiente nota marginal en el f. $47^{\mathrm{r}}$ : Grecus inquit inferens purpuram.

${ }^{53}$ El patrimonio antiguo de la actual biblioteca está constituido por los distintos fondos de las bibliotecas de los claustros benedictinos y capuchinos de Monreale y del benedictino de San Martino delle Scale, como resultado de la ley de suspensión de las corporaciones religiosas de 1866 (Schirò 1992; Ferraro 2011, p.74).

${ }^{54}$ No aparece en Tarallo (1835) ni en Schirò (1992). El primero en dar cuenta es Garufi (1902, pp.XIX, 231 $\left.\mathrm{n}^{\circ} 14\right)$ pero sin indicar procedencia. Aparece en el actual Elenco dei Manoscritti.

${ }^{55}$ Vivió en los monasterios de Cessena y en Peruggia pero no se ha constatado su paso por Florencia.

${ }^{56}$ Información ofrecida por Ignazia Ferraro en comunicación privada.
} 
serie de polémicas centradas en aspectos filosóficos, filológicos y políticos que se sitúan en la base del pensamiento político y social durante los siglos XV y XVI.

A partir de los datos ofrecidos se ha comprobado que desde el punto de vista codicológico, el manuscrito monrealense es un ejemplo de la conjunción de escritura humanística redonda y la iluminación a 'bianchi girari' de origen florentino de la primera veintena del siglo XV. De las características de su escritura y de su decoración se puede concluir que la copia se llevó a cabo en el ámbito de producción de Niccolò Niccoli y Poggio Bracciolini, impulsores de la renovación caligráfica y ornamental durante los primeros años del siglo XV. Aunque no podemos precisar el copista, sabemos que Bruni encargó a su amigo Niccoli una reescritura íntegra de la obra y podría establecerse que la copia monrealense derivase de un apógrafo del propio Niccoli.

En fin, desde el punto de vista filológico es el único testimonio que contiene intervenciones autógrafas con declaración expresa del propio Leonardo Bruni, lo que le sitúa en un lugar privilegiado para el estudio del modus operandi del autor: para conocer mejor el usus scribendi, y para determinar la labor de revisión tanto a nivel del cuidado en la corrección de la copia como a nivel crítico-textual. Bruni nos brinda en sus anotaciones marginales, por un lado, variantes propias de la emendatio ope codicum, seguramente a partir de su propia copia de autor y de otros códices griegos, distintos al / a los que le sirvieron como modelo para su traducción, y por otro lado, variantes que declaran la voluntad ante una determinada redacción. El hecho de que los marginalia referidos a la labor traductora se repitan en más de un códice con redacción similar pero no idéntica indica que en el de Monreale el autor intervino de forma directa y que en el resto ejerció, si no de su propia mano, una revisión también directa. Es, pues, un testimonio de innegable valor por tratarse de un autógrafo pero, a la vez, no puede ser determinante para todo el texto, dado que no fue corregido íntegramente, tal y como reconoce el propio Bruni.

\section{REFERENCIAS BIBLIOGRÁFICAS}

BAndini, M. (2004), «Lo Ierone di Senofonte nel Quattrocento. Leonardo Bruni e Antonio da Pescia», Res publica litterarum 28, 108-123.

BANDINI, M. (2007), «Il Tyrannus di Leonardo Bruni: note su tradizione e fortuna», en M. CoRTESI (ed.), Tradurre dal greco in età umanistica. Metodi e strumenti. Atti del Seminario di studio. Firenze, Certosa del Galluzzo, 9 settembre 2005, Florencia, SISMEL-Edizioni del Galluzo, pp.35-44.

Baron, H. (ed.) (1928), Leonardo Bruni Aretino. Humanistisch-Philosophische Schriften mit einer Chronologie seiner Werke und Briefe, Leipzig (reimp. Wiesbaden 1969), Teubner.

Baron, H. (1968), From Petrarch to Leonardo Bruni: Studies in humanistic and political literature, Chicago-Londres, Chicago University Press.

Bernasconi Reusser, M. (2000), «Inchiostri. La tecnica PIXE applicata al testo e alla decorazione di alcuni manoscritti italiani dal XII al XV secolo», Quinio 2, 45-61.

BERNASCONI REUSSER et al. (1993), "Analyse des couleurs dans un groupe de manscrits enluminés du XII ${ }^{\mathrm{e}}$ siècle avec l'emploi de la technique PIXE» en Ancient and medieval book 
materials and techiques (Erice, 18-25 september 1992), Ciudad del Vaticano, Biblioteca Apostolica Vaticana, pp.57-101.

Berti, E. (1995), «A proposito di alcuni codici greci in relazione con Manuele Crisolora e con Leonardo Bruni», Studi classici e orientali 45, 281-299.

BiancA, C. (2002), «Traduzioni interlineari dal greco nel circolo del Salutati: Jacopo Angeli, Niccolò Niccoli, Leonardo Bruni», en R. MAISANO - A. Rollo (edd.), Manuele Crisolora e il ritorno del greco in Occidente. Atti del Convegno Internazionale (Napoli, 26-29 giugno 1997), Nápoles, Istituto Universitario Orientale, pp.133-150.

Billanovich, G. (1981), «Alle origini della scrittura umanistica: Padova 1261-Firenze 1397», en Miscellanea Augusto Campana, Padua, Antenore, pp.125-140.

BirkenmaJer, A. (1922), «Der Streit des Alfonso von Cartagena mit Leonardo Bruni Aretino», Vermische Untersuchungen zur Geschichte der mittelalterlichen Philosophie, Múnich, Beiträge zur Geschichte der Philosophie des Mittelalters 20.5, pp.129-210.

Brams, J. (2009), «Riscoperta di Aristotele in Occidente» en I. BIFFI - C. MARABelli (edd.), La nuova razionalità. XIII secolo, Milán, Jaca Book, pp.59-126.

Bywater, I. (1894 [1988]), Aristotelis Ethica Nicomachea, Oxford, Bibliotheca Oxoniensis.

Blum, R. (1951), La biblioteca della Badia fiorentina e i codici di Antonio Corbinelli, Ciudad del Vaticano, Biblioteca Apostolica Vaticana.

CAsamassima, E. (1974), «Literulae latinae. Nota paleografica», en S. CAROTI - S. ZAMPONI, Lo scrittoio di Bartolomeo Fonzio, Milán, Il Polifilo («Documenti sulle arti del libro», X), pp.9-33.

Ceccanti, M. (1993-1996), «Proposte per la storia dei primi codici umanistici a bianchi girari», Miniatura: studi di storia dell'illustrazione e decorazione del libro 5-6, 11-16.

Chiesa, P. - PINELli, L. (edd.) (1994), Gli autografi medievali. Problemi paleografici e filologici. Atti del Convegno di Studi della Fondazione Ezio Franceschini. Erice 25 settembre-2 ottobre 1990, Spoletto.

Chiesa, P. (2002), Elementi di critica testuale, Bolonia, Pàtron (reimpr. 2011).

Ciccuto, M. (2007), «Poggio "conoscitore” fra codici antichi illustrati», Humanistica. An International Journal of Early Renaissance Studies 2, 55-63.

CORTESI, M. - FIASCHI, S. (2008) (edd.), Repertorio delle traduzioni umanistiche a stampa: secoli $X V-X V I, 2$ vols., Florencia, SISMEL-Edizioni del Galluzzo.

De Robertis, T. (1998), «Motivi classici nella scrittura del primo Quattrocento» en P. CASTELLI (ed.), L’ideale classico a Ferrara e in Italia nel Rinascimento, Florencia, Olschki, pp.65-79.

De Robertis, T. (2006), «I percorsi dell'imitazione. Esperimenti di Littera Antiqua in codici fiorentini del primo Quattrocento», en C. TRistano - M. CALleri - L. MAgionami (edd.), I luoghi dello scrivere da Francesco Petrarca agli albori dell'Ètà Moderna. Atti del Convegno internazionale di studio dell'Associazione italiana dei Paleografi e Diplomatisti. Arezzo (8-10 ottobre 2003), Spoleto, Centro Italiano di Studi sull'Alto Medioevo.

De Robertis, T. (2010), «Salutati tra scrittura gotica e littera antiqua», en C. BiAnCA (ed.), Coluccio Salutati e l'invenzione dell'Umanesimo. Atti del Convegno Internazionale di Studi. Firenze 29-31 ottobre 2008, Roma, Edizioni di Storia e Letteratura, pp.369-395.

FERrARo, I. (2011), «Una singolare mostra in una antica biblioteca: Parva Naturalia, annotazioni da Aristotele», catálogo de la exposición Parva Naturalia, annotazioni da Aristotele. Rassegna di Libri di Artisti Siciliani a cura di Antonina Greco. Biblioteca Comunale Santa Maria La Nuova. Fondo Antico. Complesso Monumentale Guglielmo II. 18-20 novembre 2011, Monreale, pp.74-87. 
Franceschini, E. (1955), «Leonardo Bruni e il "vetus interpres" dell'Etica a Nicomaco», en G. C. SAnsoni (ed.), Medioevo e Rinascimento. Studi in onore di Bruno Nardi, 2 vols., Florencia, vol. I, pp.297-319.

Gasparri, F. (1994), «Authenticité des autograhes», en ChiesA - Pinelli (edd.), pp.3-22.

Gauthier, R.A. - Jolif, J.Y. (1970²), L’Éthique a Nicomaque, Lovaina-París, Institut Supérieur de Philosophie de l'Université de Louvain.

GAUthIER, R.A. (ed.) (1973), Aristoteles Latinus. Ethica Nicomachea. Translatio Roberti Grosseteste Lincolniensis sive Liber Ethicorum. B. Recensio recognita, Leiden, E. J. Brill.

GENTILE, S. (1992), Firenze e la scoperta dell'America: umanesimo e geografia nel '400 fiorentino, Florencia, Olschki.

Giustiniani, V.R. (1964), «Il testamento di Leonardo Bruni», Rinascimento n.s 4, 259-264.

González Rolán, T. - Moreno Hernández, A.- Saquero SuÁrez-Somonte P. (2000), Humanismo y Teoría de la Traducción en España e Italia en la primera mitad del siglo XV. Edición y estudio de la Controversia Alphonsiana (Alfonso de Cartagena vs. L. Bruni y P. Cándido Decembrio), Madrid, Ediciones Clásicas.

Griggio, C. (1986), «Due lettere inedite del Bruni al Salutati e a Francesco Barbaro», Rinascimento n.s. 26, 27-50.

Gualdo Rosa, L. (ed.) (1980), Francesco Paolo Luiso. Studi su l'epistolario di Leonardo Bru$n i$, Roma, Istituto Storico Italiano per il Medio Evo.

Hankins, J. (1997), Repertorium Brunianum: a Critical Guide to the Writings of Leonardo Bruni. Volume I. Handlist of Manuscripts, Roma, Istituto Storico Italiano per il Medio Evo.

HANKINS, J. (2001), «Notes on Leonardo Bruni's Translation of The Nicomachean Ethics and its Reception in the Fifteenth Century», en J. HAMESSE (ed.), Les traducteurs au travail. Leurs manuscrits et leurs méthodes. Actes du colloque international organisé par le "Ettore Majorana Centre for Scientific Culture” (Erice, 30 septembre - 6 octobre 1999), Turnhout, Brepols, pp.427-447.

Hankins, J. (2003), «The Ethics controversy», en J. Hankins (ed.), Humanism and Platonism in the Italian Renaissance, Roma, Edizioni di Storia e Letteratura, pp.193-239.

HARLFINGER, D. (1980), «Einge Grundzüge der Aristoteles-Überlieferung», en D. HARLFINGER (ed.), Griechische kodikologie und textuberlieferung, Darmstadt, Wissenschaftliche Buchgesellschaft, pp.447-483.

Hart, H. (ed.) (1984a), Poggio Bracciolini. Lettere. I. Lettere a Niccolò Niccoli, Florencia, Olschki.

Hart, H. (1984b), Poggio Bracciolini. Lettere. II. Epistolarum familiarium libri, Florencia, Olschki.

Kristeller, P.O. (1963-1992), Iter Italicum. A Finding List of Uncatalogued or Incompletely Catalogued Humanistic Manuscripts of The Renaissance in Italian and Other Libraries, 6 vols., Londres-Leiden, Brill.

KRISTEller, P.O. (1996), «The Editing of Fifteenth-Century Texts. Tasks and Problems» en Studies in Renaissance Thought and Letters, vol. 4, Roma, Edizioni di Storia e Letteratua, pp.457-463.

LINES, D. (2002), Aristotle's Ethics in the Italian Renaissance (ca. 1300-1650): the Universities and the problem of moral education, Leiden-Boston, Brill.

MARCHESI, C. (1904), L'Etica nicomachea nella tradizione latina medievale: documenti e appunti, Messina, Trimarchi. 
De la Mare, A.C. (1973), The Handwriting of Italian Humanist. 1.1: Francesco Petrarca, Giovanni Boccaccio, Coluccio Salutati, Niccolò Niccoli, Poggio Bracciolini, Bartolomeo Aragazzi of Montepulciano, Sozomeno of Pistoia, Giorgio Antonio Vespucci, Oxford, Association Internationale de Bibliophilie.

De la Mare, A.C. (1977), «Humanistic Script: the first ten Years», en F. Kraft - D. WutTKe, Boppard, H. Boldt Verlag (edd.), Das Verhältnis der Humanisten zum Buch, Bonn-Bad, Deutsche Forschungsgemeinschaft Kommission für Humanismusforschung, Mitteilung IV, pp.89-110.

De La Mare, A.C. - Thomson, F.S. (1973), «Poggio’s Earliest Manuscript?», Italia Medioevale e Umanistica 16, 171-196.

Mehus, L. (ed.) (1741), Leonardi Bruni Arretini Epistolarum libri VIII, Florencia.

Morrás, M. (2002), «El debate entre Leonardo Bruni y Alonso de Cartagena: las razones de una polémica», Quaderns. Revista de traduccció 7, 33-57.

PÄсHT, O. (1957), «Notes and Observations on the Origin of Humanistic Book Decoration», en D.J. Gordon (ed.), Fritz Saxl (1890-1948). A Volume of Memorial Essays from his Friends in England, Londres, Nelson \& Sons, pp.184-194.

Perosa, A (2000), «Critica congetturale e testi umanistici» en P. Viti (ed.), Alessandro Perosa. Studi di filologia umanistica II. Quattrocento fiorentino, Roma, Edizioni di Storia e Letteratura, pp.11-40.

PinTor, F. (1902), La libreria di Cosimo de' Medici nel 1418, Florencia, S. Landi.

Pomaro, G., «Fila traversariane. I codici di Lattanzio», en G. C. GARFAGNini (ed.), Ambrogio Traversari nel VI centenario della nascita: Convegno internazionale di studi (Camaldoli-Firenze, 15-18 settembre 1986), Florencia, Olschki, pp.235-285.

ReEve, M.D. (1994), «Errori in autografi», en ChiESA - Pinelli (edd.), pp.37-60.

Rizzo, S. (1984²), Il lessico filologico degli umanisti, Roma, Edizioni di Storia e Letteratura.

Rollo, A. (2004), «Sulle tracce di Antonio Corbinelli», Studi medievali e umanistici 2, 25 95.

Scarcia Piacentini, P. (1981), «La tradizione laudense di Cicerone ed un inesplorato manoscritto della Biblioteca Vaticana (Vat. Lat. 3287)», Revue d'Histoire des Textes 12, 123-146.

Schirò, G. (1992), Le biblioteche di Monreale: la Biblioteca del Seminario e la Biblioteca Comunale, Palermo, Sellerio.

Schмiтt, Сн. B. (1983), Aristotle and the Renaissance, Cambridge-Mass, Harvard University Press (Trad. española, Aristóteles y el Renacimiento, León 2004).

Suseminl, F. (ed.) (1882), Aristotelis Ethica Nicomachea, Lipsiae, Teubneri.

Tarallo, G. Batt. (1835), Catalogo dell'Ediz. del sec. XV dei Cod. della Biblioteca dei PP. Benedettini Cassinesi di Monreale, Palermo.

Trendelenburg, A. (1867), Historische Beiträge zur Philosophie, vol. 3, Berlin, G. Bethge.

Ullman, B.L., The Origin and Development of Humanistic Script, Roma 1960, Edizioni di Storia e Letteratura.

Viti, P. (1992), «Preliminari per uno studio sulla grafia di Leonardo Bruni» en P. ViTi, Leonardo Bruni e Firenze: studi sulle lettere pubbliche e private, Roma, Bulzoni, pp.275307.

ViTi, P. (1993), «Un'antologia di opere di Leonardo Bruni. Il manoscritto laurenziano 52,5», Rinascimento s. 2, 33, 157-161. 
VITI, P. (1994), «Un nuovo codice con postille di Leonardo Bruni», Lettere italiane 36, 420424.

Venier, M. (ed.) (2011), Platonis Gorgias. Leonardo Aretino interprete, Florencia, SISMELEdizione del Galluzzo.

ZACCARIA, R. (1998), «Un autografo cancelleresco di Coluccio Salutati», en T. DE RoBertis - G. SAvino (edd.), Tra libri e carte. Studi in onore di Luciana Mosiici, Florencia, Franco Cesati Editore, pp.543-548. 